\title{
Adaptación de metodologías docentes para la enseñanza de problemas en aula informática con el objetivo de formar en competencias de nivel de Máster
}

\section{José M. Gozálvez Zafrilla, Asunción Santafé Moros, Javier Navarro Laboulais}

Equipo de Innovación y Calidad Educativa ASEI (Aplicación de la Simulación en la Enseñanza de la Ingeniería), Departamento de Ingeniería Química y Nuclear, Universitat Politècnica de Valencia, Valencia, España, jmgz@iqn.upv.es, assanmo@iqn.upv.es, jnavarla@iqn.upv.es

\begin{abstract}
This work analyzes the adaptation and development of teaching methodologies for problem solving strategies in the subject "Advanced Unit Operations" of the Master in Chemical Engineering for developing transversal key competences. As starting point, it has been used the teaching methodology developed in the former curriculum adapted to the new one. The adaptation was performed through two different ways. On the one hand, problems of open response supported by mathematical software were included. On the other hand, new computational resources, like simulation and design software, were introduced to make the student easier the exploration of different process alternatives. The inclusion of these problems implied changes in the evaluation procedure. The effects of the methodological changes performed are shown in relation with the acquired competences by the students and with the necessary changes in the evaluation procedure. A survey among the students shows a positive opinion in relation to the methodological adaptations performed.
\end{abstract}

Keywords: problem solving, transversal competences, active methodologies, software resources, simulation

\footnotetext{
Resumen

En este trabajo se analiza la adaptación y desarrollo de metodologías docentes para el aprendizaje de la resolución de problemas en la asignatura del Máster de Ingeniería Química "Operaciones de Separación Avanzadas" con el objetivo de desarrollar competencias transversales. Como punto de partida se considera la metodología que desarrollamos para las clases de problemas del plan de estudios previo. La adaptación se realizó a través de
} 
Adaptación de metodologías docentes para la enseñanza de problemas en aula informática con el objetivo de formar en competencias de nivel de Máster

dos vías diferentes. Por una parte, se realizaron cambios en el tipo de problemas propuestos y en la forma de evaluación, trabajándose con problemas de tipo más abierto apoyados por software matemático. Por otra parte, se introdujeron nuevos recursos como programas de simulación y de diseño de procesos con el fin de facilitar al alumno la posibilidad de explorar alternativas. Los efectos de estos cambios se indican en este artículo relacionándolos con las competencias que favorecen y con los cambios en el sistema de evaluación necesarios. El análisis de los resultados de una encuesta mostró una opinión favorable hacia las adaptaciones metodológicas realizadas.

Palabras clave: resolución de problemas, competencias transversales, metodologías activas, recursos informáticos, simulación.

\section{Introducción}

La materia "Operaciones de Separación” representa una parte importante del currículo del Ingeniero Químico. Tanto es así que el Manual del Ingeniero Químico (Perry, 2014), obra de referencia de la Ingeniería Química le dedica un $40 \%$ de sus contenidos. Por ello, la asignatura de Grado "Operaciones de Separación" (4.5 créditos) se complementó en el Máster de Ingeniería Química con la asignatura “Operaciones de Separación Avanzadas” de 6 créditos.

Dentro de la materia resulta fundamental la realización de problemas donde los alumnos aprenden a diseñar y calcular los equipos con el objetivo de lograr una determinada separación de los componentes de una corriente de proceso. En nuestra experiencia con la titulación previa, los problemas resultan difíciles a los alumnos por los siguientes factores:

- Requieren un sólido conocimiento teórico de los fenómenos físicos y del modo de funcionamiento del equipo de separación.

- El número de variables es alto y la notación abundante.

- Involucran un gran número de pasos, requiriendo algunos de ellos el apoyo de métodos gráficos para su comprensión.

- Se necesita aplicar métodos matemáticos y algoritmos diversos.

La conjunción de todos estos factores representa una gran dificultad para el alumno que se detiene en los detalles perdiendo la visión global del problema. Esto justifica la conveniencia de usar adecuadamente herramientas de cálculo para disminuir el esfuerzo del alumno. Por ello, en planes anteriores y en la asignatura de Grado utilizamos una metodología de enseñanza de problemas que emplea el software matemático Mathcad® 
Esta metodología ha demostrado su eficacia para facilitar la comprensión de los cálculos, permitiendo la resolución de problemas más complejos y, por tanto, más realistas.

En el diseño de la nueva asignatura del Máster se consideró conveniente, además de aportar nuevos contenidos, desarrollar competencias transversales adaptadas a este nivel. Ello requirió adaptar la metodología docente de enseñanza de problemas, aunque sin dejar de utilizar Mathcad como herramienta de apoyo principal. Como veremos, la propia realización de problemas implica desarrollar en alto grado las competencias transversales importantes. La introducción de herramientas de software desarrolla competencias instrumentales específicas en sí misma y otras competencias pueden desarrollarse gracias a la disminución del tiempo necesario para realizar los problemas.

\section{Objetivos}

El objetivo principal de las acciones realizadas fue adaptar las metodologías de enseñanza de problemas utilizadas en planes anteriores y en el nivel de Grado para desarrollar al máximo competencias fundamentales de nivel de Máster.

De esta forma se tuvieron que cubrir los siguientes objetivos parciales:

- Realizar un análisis de la situación previa así como de las competencias específicas de los titulados de nivel de Máster.

- Adaptar el enfoque de la docencia de los problemas, de acuerdo con los objetivos competenciales y las condiciones del entorno, con el fin de favorecer la adquisición de competencias transversales en el caso de la asignatura de Máster.

- Adaptar las metodologías de evaluación al tipo de docencia.

- Analizar los efectos de los cambios en el primer curso de implantación, considerando la percepción de los alumnos.

\section{Desarrollo de la innovación}

\subsection{Análisis de la situación previa y del contexto actual}

\subsubsection{Situación previa}

En nivel de Grado, el aprendizaje de la resolución de problemas se realiza en aula informática de manera individual empleando el entorno de cálculo matemático Mathcad®. La elección de Mathcad como software matemático de apoyo vino determinada por su

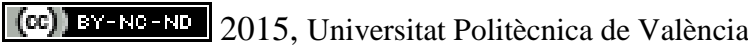


Adaptación de metodologías docentes para la enseñanza de problemas en aula informática con el objetivo de formar en competencias de nivel de Máster

facilidad de uso y por utilizar una visualización cercana a la notación matemática convencional que facilita el estudio de los problemas. Debido a la importancia que suponen los cálculos de diseño de las unidades, se dedica un 50\% de los créditos a prácticas donde se trabaja este aspecto. En ellas, el alumno, además de aprender a realizar el diseño de unidades de separación, aprende a estudiar el efecto de los parámetros de diseño, empleando los cálculos que ha aprendido, y reforzando con ello sus conocimientos teóricos. Como material de apoyo, hemos desarrollado ejemplos de cálculo de las unidades más comunes (Gozálvez et al. 2012, Santafé el al. 2013), así como de objetos de aprendizaje en forma de laboratorios virtuales de simulación con los que también puede explorar el efecto de los parámetros (Gozálvez 2008, Santafé et al. 2009).

\subsubsection{Contexto de la nueva asignatura de Máster}

En la nueva asignatura concurren otras circunstancias que han obligado a realizar ciertos cambios metodológicos:

- $\quad$ El porcentaje de créditos de prácticas de laboratorio (20\%) es bajo.

- Los cálculos revisten complejidad. Se resuelven situaciones multicomponente.

- El alumno posee conocimientos previos de la materia, por lo que ciertos procedimientos sólo deben repasarse brevemente.

- Es aconsejable desarrollar más ciertas competencias transversales, p.e., es de esperar un mayor énfasis en la toma de decisiones y en la capacidad de considerar aspectos globales de tipo económico o medioambiental.

\subsection{Análisis de competencias}

La Tabla 1 recoge las trece dimensiones competenciales consideradas en la UPV (VECA, 2014). De una lectura de la definición de las competencias expuestas se desprende cuáles son las que intervienen siempre en una clase de resolución de problemas de la asignatura. Estas competencias son consideradas como objetivos principales, siendo la competencia DC3 la más importante:

- DC1 Comprensión e integración.

- $\quad$ DC2 Aplicación y pensamiento práctico.

- DC3 Análisis y resolución de problemas. 
Tabla 1. Dimensiones competenciales UPV

DC1 Comprensión e integración

DC2 Aplicación y pensamiento práctico

DC3 Análisis y resolución de problemas

DC4 Innovación, creatividad y emprendimiento

DC5 Diseño y proyecto

DC6 Trabajo en equipo y liderazgo

DC7 Responsabilidad ética, medioambiental y profesional

DC8 Comunicación efectiva

DC9 Pensamiento crítico

DC10 Conocimiento de problemas contemporáneos

DC11 Aprendizaje permanente

DC12 Planificación y gestión del tiempo

DC13 Instrumental específica
Demostrar la comprensión e integración del conocimiento tanto de la propia especialización como en otros contextos más amplios.

Aplicar los conocimientos a la práctica, atendiendo a la información disponible, y estableciendo el proceso a seguir para alcanzar los objetivos con eficacia y eficiencia.

Analizar y resolver problemas de forma efectiva, identificando y definiendo los elementos significativos que lo constituyen.

Innovar para responder satisfactoriamente y de forma original a las necesidades y demandas personales, organizativas y sociales con actitud emprendedora.

Diseñar, dirigir y evaluar una idea de manera eficaz hasta concretarla en un proyecto.

Trabajar y liderar equipos de forma efectiva para la consecución de objetivos comunes, contribuyendo al desarrollo personal y profesional de los mismos.

Actuar con responsabilidad ética y profesional ante uno mismo y los demás.

Comunicarse de manera efectiva, tanto de forma oral como escrita, utilizando adecuadamente los recursos necesarios y adaptándose a las características de la situación y la audiencia.

Desarrollar un pensamiento crítico interesándose por los fundamentos en los que se asientan las ideas, acciones y juicios, tanto propios como ajenos.

Conocimiento de los problemas contemporáneos.

Utilizar el aprendizaje de manera estratégica, autónoma y flexible, a lo largo de toda la vida, en función del objetivo perseguido.

Planificar adecuadamente el tiempo disponible y programar las actividades necesarias para alcanzar los objetivos, tanto académicos-profesionales como personales.

Capacidad para utilizar las técnicas, las habilidades y las herramientas actualizadas necesarias para la práctica de la profesión. 
Adaptación de metodologías docentes para la enseñanza de problemas en aula informática con el objetivo de formar en competencias de nivel de Máster

La propia implantación de la metodología de resolución de problemas permitió plantear unos objetivos competenciales adicionales asociados a las metodologías docentes que se iban a utilizar:

- DC5 Diseño y proyecto

- DC6 Trabajo en equipo y liderazgo

- DC13 Instrumental específica

La competencia DC5 está asociada al tipo de problemas utilizado, en los que se emplean cálculos para hacer un diseño concreto. La DC6 se podrá desarrollar si se permite trabajar por parejas o en grupos. La DC13 estaría asociada al aprendizaje del software empleado.

Adicionalmente, parece interesante incluir entre los objetivos la siguiente competencia:

- DC7 Responsabilidad ética, medioambiental y profesional

Esta competencia puede desarrollarse en ciertos problemas de la asignatura, donde se estudian operaciones de separación que pueden tener tanto una utilidad industrial como medioambiental.

La misma metodología de trabajo en la clase de problemas permite subsidiariamente el desarrollo de la mayor de las competencias restantes, y se va aprovechar este hecho para conseguir otros objetivos competenciales (Tabla 2).

\subsection{Adaptaciones metodológicas}

En el apartado 2.1 ya se ha comentado la metodología docente seguida en planes anteriores y en gran parte en el Grado actual, la cual se toma como punto de partida para el Máster. Algunas operaciones de separación o la integración en un proceso químico dan lugar a problemas de gran complejidad matemática que en ocasiones son percibidos por los alumnos como insuperables. La utilización de una herramienta como Mathcad permite resolver estos problemas matemáticos dejando más tiempo para orientar el aprendizaje del alumno hacia competencias más específicas de la titulación Por ello, el uso de Mathcad se mantiene como elemento central. No obstante, para permitir el desarrollo de nuevas competencias se procedió a las siguientes adaptaciones de la metodología:

- Redefinición de los enunciados para lograr problemas más abiertos

- Cambios en la metodología de uso del software matemático

- Introducción de herramientas de simulación adicionales

- Cambios en la dinámica de la clase: actuación del profesor y participantes

- Cambios en el sistema de evaluación 
Tabla 2. Relevancia de la competencia para los objetivos de aprendizaje buscados en la clase de resolución de problemas

\begin{tabular}{llc}
\hline & \multicolumn{1}{c}{ Dimensión competencial UPV } & Relevancia \\
\hline DC1 & Comprensión e integración & Alta \\
DC2 & Aplicación y pensamiento práctico & Alta \\
DC3 & Análisis y resolución de problemas & Muy alta \\
DC4 & Innovación, creatividad y emprendimiento & \\
DC5 & Diseño y proyecto & Baja \\
DC6 & Trabajo en equipo y liderazgo & Baja \\
DC7 & Responsabilidad ética, medioambiental y profesional & Media \\
DC8. & Comunicación efectiva & Media \\
DC9 & Pensamiento crítico & Medio \\
DC10 & Conocimiento de problemas contemporáneos & Baja \\
DC11 & Aprendizaje permanente & \\
DC12 & Planificación y gestión del tiempo & \\
DC13 & Instrumental específica & Alta \\
\hline
\end{tabular}

\subsubsection{Redefinición de los enunciados para lograr problemas más abiertos}

Una forma eficaz de introducir otras competencias transversales es replantear los enunciados de los problemas para que la solución no esté cerrada favoreciendo así la reflexión.

Evidentemente, esto no se puede aplicar en todos los problemas, pues el alumno también debe aprender la realización de cálculos cortos específicos de cada operación. Estos cálculos se identificaron y se asignaron a las clases de teoría y de práctica de aula impartidas en un único grupo de aula. En estos problemas se siguió un enfoque tradicional, en el que el alumno, después de recibir los conceptos teóricos necesarios para realizar las distintas partes del cálculo, es guiado en el cálculo de la unidad (enfoque bottom-up).

En contrapartida, los problemas más extensos presentan mayores oportunidades para el desarrollo de competencias. Estos fueron reservados para las clases informáticas en las que los alumnos al ser divididos en tres grupos de aula podían recibir mayor atención por parte del profesor.

\section{(c)) EY-NC-ND 2015, Universitat Politècnica de València}


Adaptación de metodologías docentes para la enseñanza de problemas en aula informática con el objetivo de formar en competencias de nivel de Máster

En los problemas se planteó, un enunciado más general, utilizando algunas de las siguientes modificaciones:

- No pedir el cálculo de una unidad concreta, sino pedir la selección entre varias alternativas.

- Proporcionar información en exceso, para que el alumno seleccione los datos relevantes.

- No proporcionar datos relevantes que el alumno deberá suponer o buscar en red.

- Proponer estudiar el efecto de un parámetro, de manera que el alumno lo debe justificar de acuerdo con la teoría.

- Solicitar una decisión basada en el criterio propio del alumno, siendo interesante que incluya una decisión económica y medioambiental.

Obsérvese que se busca que el alumno sea capaz de particularizar para resolver situaciones específicas (enfoque top-down) las cuales deberá comparar y analizar para obtener información. Además, en casos particulares, se pueden proporcionar reglas de apoyo basadas en la experiencia, pues tal y como indican (Molero, M. y Salvador, A., 2014), la resolución de problemas es el contexto adecuado para ejercitar el método heurístico. Indicamos además que esto es cierto especialmente en los problemas de diseño. En definitiva, el objetivo no es tanto aprender un cálculo, como saber modificar un esquema de cálculo y crear conocimiento a partir de él.

\subsubsection{Cambios en la metodología de uso del software matemático}

En los problemas de Grado, de manera similar a como se planteaba en el plan de estudios anterior, el alumno parte de un enunciado que consta de varios apartados que al seguirlos completan el cálculo de la unidad. Estos cálculos son realizados en Mathcad y se le proporcionan subrutinas de datos y de algunos cálculos repetitivos. En algunos problemas, puede incluirse un apartado final en el que se solicita algún estudio paramétrico o una reflexión sobre el cálculo de la unidad completo.

A nivel de máster, en los problemas se proporciona casi toda la estructura de un cálculo estándar excepto algunas partes específicas que requieren razonamiento o que deben modificarse para adaptar el problema a variantes solicitadas en el enunciado. Proporcionar la mayor parte de cálculos es imprescindible para poder disponer de tiempo para que el alumno pueda utilizarlos para el análisis. Se puede pensar que el alumno no llega a aprender a realizar el cálculo completo, pero recordemos que se aprovecha que el alumno ya ha seguido en el Grado una asignatura en la que lo hacía así, además, no es óbice para que puede exigirse el estudio de la estructura del cálculo y éste sea evaluado en el examen. 


\subsubsection{Introducción de herramientas de simulación adicionales}

Los simuladores de procesos son utilizados en situaciones en las que la resolución del problema mediante cálculo y programación es demasiado compleja. En nuestro caso, aprovechamos la disponibilidad del software comercial de simulación de procesos CHEMCAD ${ }^{\circledR}$, como medio de complementar los cálculos realizados con el modelo implementado en Mathcad, de forma que se puede realizar una situación más compleja, p.e. un cálculo multicomponente o no isotermo. Para el caso de determinadas operaciones hemos utilizado también software gratuito proporcionado por empresas, p.e. el programa ROSA® para cálculos de sistemas de membranas.

\subsubsection{Cambios en la dinámica de la clase}

Debido al mayor número de situaciones a estudiar y a la necesidad de discutir más los resultados, se consideró que la mejor forma de trabajar con los nuevos enunciados era por parejas, pudiéndose aumentar el tamaño de grupo a 3 o 4 dependiendo de la dificultad de la práctica. De esta forma se permitía además desarrollar la competencia de trabajo en equipo.

La actuación del profesor consiste en su mayor parte en ayudar a relacionar los materiales de aprendizaje con los conocimientos previos de los alumnos. En el caso en el que el alumno no sepa cómo seguir, puede remitirle a recursos disponibles en la web.

\subsubsection{Sistema de evaluación}

Se asignó un 30\% de la nota de curso a los problemas realizados en aula informática. A la hora de calificar se evaluaba no sólo el resultado, sino también la discusión sobre lo obtenido. Para facilitar la corrección se creó una plantilla de fichero Excel con macros, donde en cada hoja debe resolverse un apartado específico mediante inclusión de datos calculados, elaboración de una zona gráfica y análisis realizado en un cuadro de texto cuyo contenido máximo puede delimitarse desde Visual Basic.

\section{Resultados}

Durante el curso 2014-2015 se realizaron seis actividades de práctica informática. Con ellas se consigue cubrir las operaciones principales estudiadas en la asignatura. El objetivo mínimo de las distintas prácticas fue de formar al alumno en los cálculos principales de la operación en cuestión, así como desarrollar como mínimo las competencias DC1, DC2, DC3, DC13 y al menos dos del resto de competencias, de manera que se cubrieron todas las competencias objetivo con el conjunto de prácticas. En aras de facilitar la comprensión, a 
Adaptación de metodologías docentes para la enseñanza de problemas en aula informática con el objetivo de formar en competencias de nivel de Máster

continuación, se utiliza una de las actividades, para mostrar en detalle cómo se logran cubrir múltiples competencias.

\subsection{Descripción de un problema adaptado}

El problema considerado es el diseño de un sistema de evaporador multiefecto, que busca obtener las dimensiones del equipo y las necesidades de vapor. Este cálculo reviste cierta complejidad por el elevado número de ecuaciones y su resolución de carácter iterativo. En el Grado de Ingeniería Química se explica el cálculo de un sistema de evaporación simple, centrando el esfuerzo en la comprensión de los fenómenos y la metodología de cálculo. El problema usado en el plan de estudios antiguo solicitaba el cálculo de una única configuración para un único compuesto. En cambio, en el problema adaptado se va a solicitar la resolución de diferentes configuraciones que pueden ser logradas conectando los efectos de diferente forma.

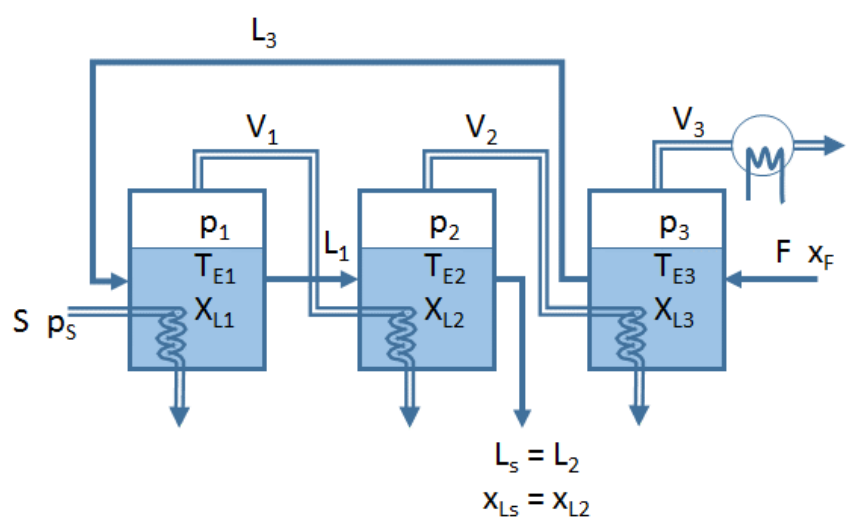

Fig. 1 Una de las posibles configuraciones de estudio

En el nuevo problema se proporciona al alumno un fichero Mathcad con diferentes zonas que pueden desplegarse donde se han interpolado o correlacionado los datos para tres compuestos distintos a concentrar (Figura 2), y que el alumno podrá seleccionar. El fichero contiene además la mayor parte de cálculos así como la subrutina de iteración necesaria, los cuales han sido explicados en teoría. No obstante, sólo se solicitan algunas partes que son claves en el cálculo, y sobretodo, aquellas que sirven para particularizar a cada situación. Por ejemplo, en la Figura 3, aparece enmarcada la zona donde el alumno definiría las ecuaciones de balance de energía necesarias, las cuales no aparecerían en el fichero de trabajo. 
Adicionalmente se solicitan al alumno diversas tareas como comparar los parámetros de diseño obtenidos para dos compuestos diferentes o seleccionar la configuración más adecuada desde el punto de vista económico o medioambiental (Figura 4). El aprendizaje se complementa trasladando los parámetros de diseño a un fichero del programa simulador donde está implementado un diagrama de flujo del proceso con el fin de estudiar el efecto de los parámetros de forma más completa (Figura 5).

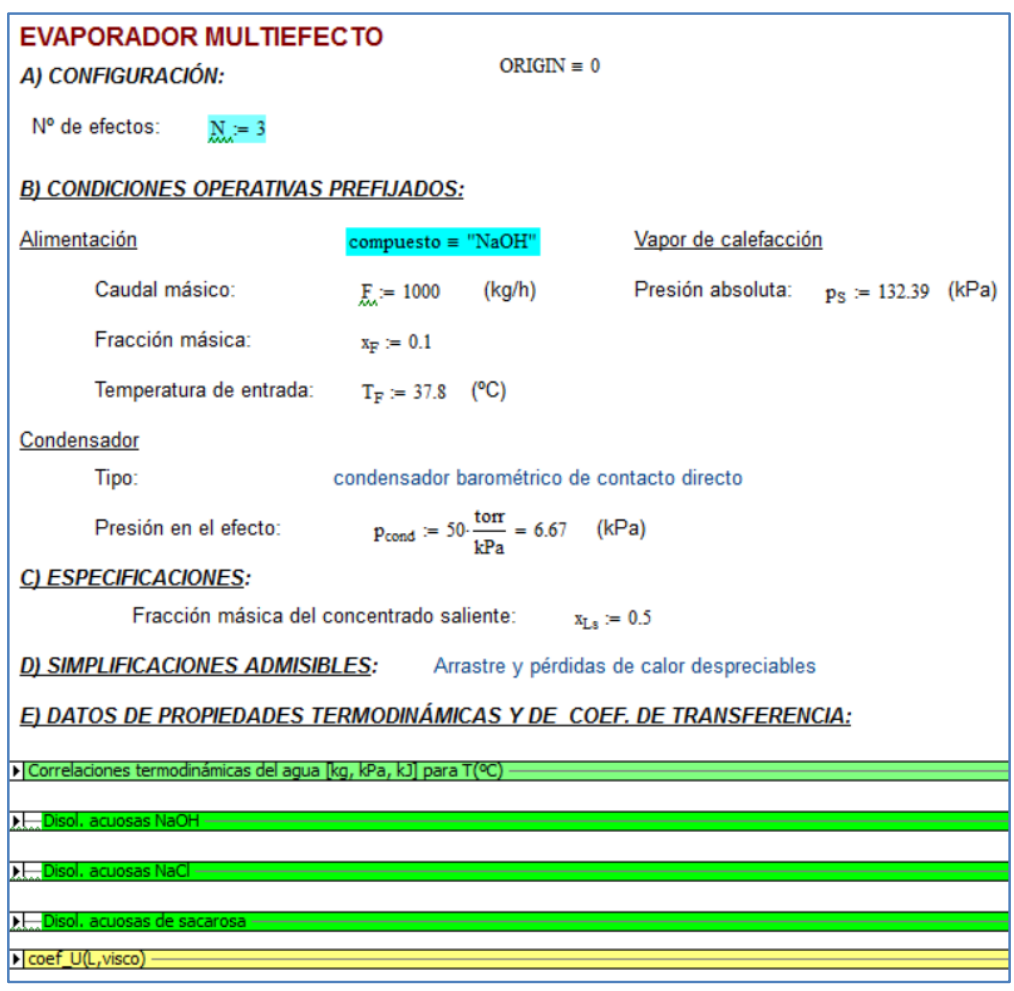

Fig. 2 Parte del fichero Mathcad que contiene las zonas de datos 
Adaptación de metodologías docentes para la enseñanza de problemas en aula informática con el objetivo de formar en competencias de nivel de Máster

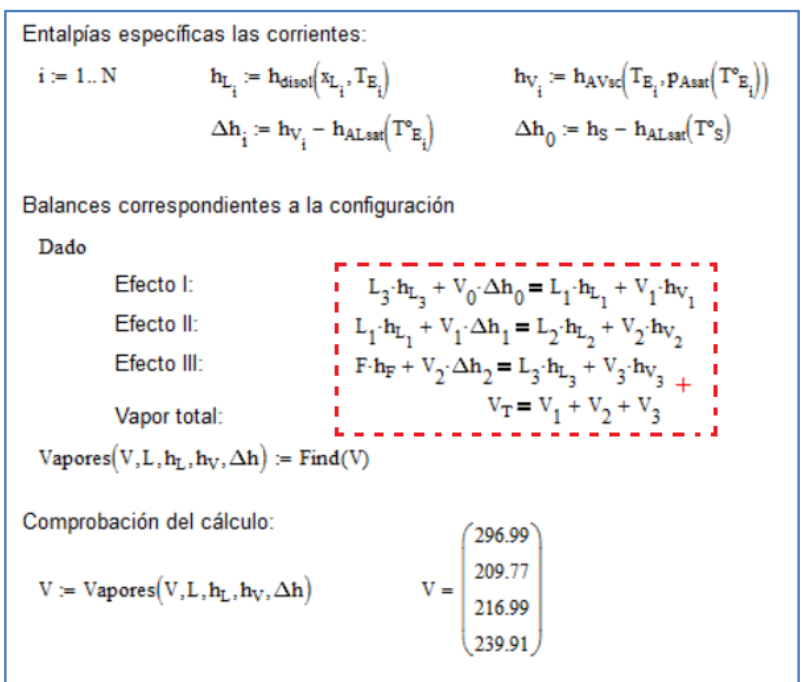

Fig. 3 Una de las zonas de Mathcad donde el alumno particulariza el cálculo para cada configuración estudiada

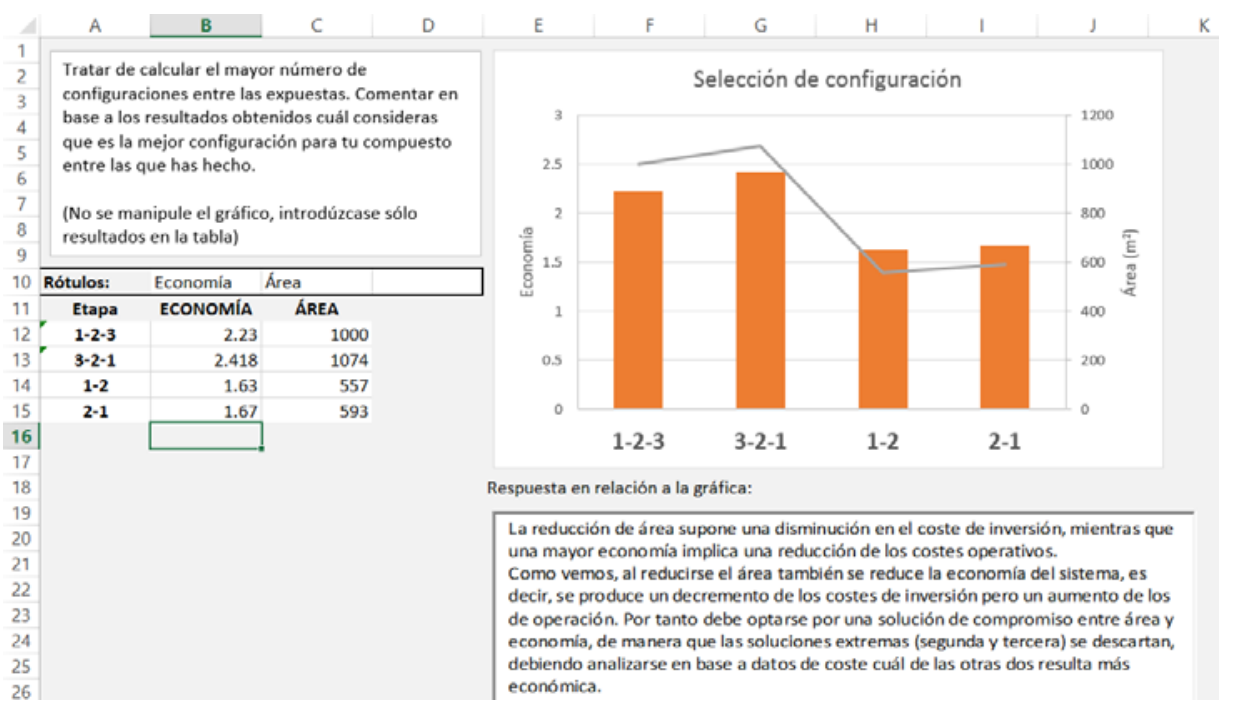

Fig. 4 Ejemplo de respuesta en una de las hojas del fichero de Excel con macros usado para la evaluación

(c)) EY-NC-ND 2015, Universitat Politècnica de València 


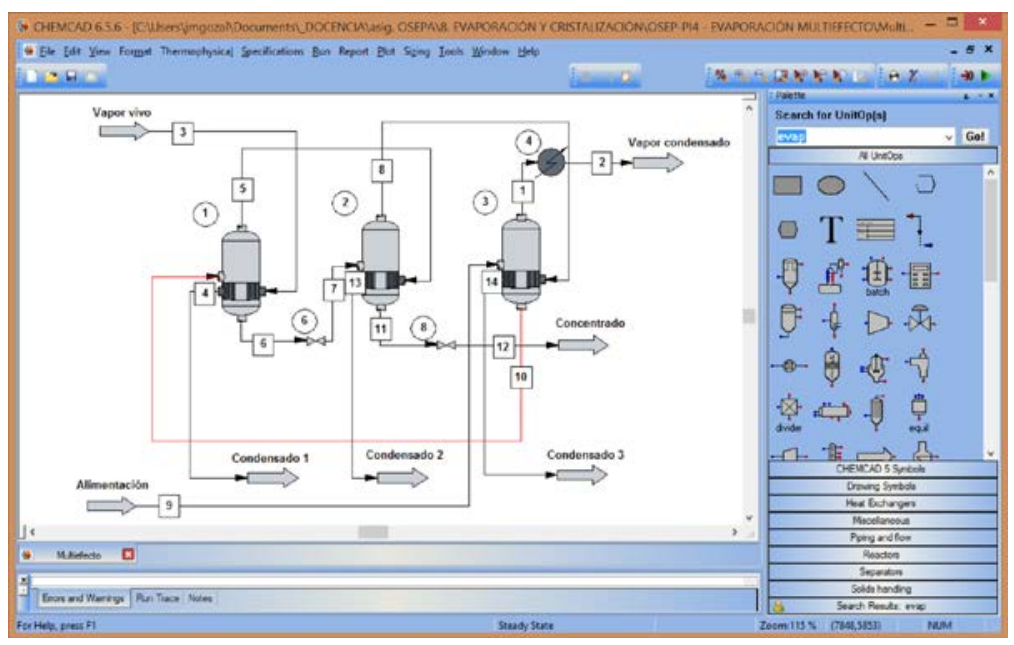

Fig. 5 Diagrama de flujo de proceso implementado en Chemcad

\subsection{Resultados de aprendizaje alcanzados}

Si se solicita la realización de los diferentes casos y un análisis de los resultados obtenidos con un esquema como el del ejemplo considerado se logra potenciar diferentes competencias, a continuación se indica cómo se favorece cada una en este problema concreto:

- DC1 Comprensión e integración:

Se compara y examinan semejanzas y diferencias entre configuraciones o sustancias a concentrar. Se analiza y sintetiza para descubrir las relaciones entre los elementos del sistema logrando nuevas configuraciones. Cuando se pregunta por qué un factor actúa de una determinada forma, se descubren razones que sustentan la conducta del equipo a través del análisis de las ecuaciones.

- DC2 Aplicación y pensamiento práctico:

El alumno recibe datos en exceso y otros los debe buscar en la red (p.e. costes) con lo cual se parece más a una situación real. Además, existe cierta incertidumbre en algunos parámetros sobre los que debe tomar una decisión.

- DC3 Análisis y resolución de problemas:

El alumno recoge en la hoja Excel la información significativa obtenida con el modelo de cálculo y el simulador de procesos, describiendo los hechos más relevantes. Debe realizar un análisis global, trabajando con aspectos 
Adaptación de metodologías docentes para la enseñanza de problemas en aula informática con el objetivo de formar en competencias de nivel de Máster

multidisciplinares como son la física del fenómeno, la síntesis de procesos y la economía.

- DC6 Trabajo en equipo y liderazgo:

Los participantes deben trabajar de forma coordinada para ser efectivos.

- DC7 Responsabilidad ética, medioambiental y profesional:

El alumno debe reflexionar sobre el consumo de energía del equipo como característica medioambiental. En otras prácticas es más patente la implicación medioambiental.

- $\quad$ DC8 Comunicación efectiva:

La respuesta dentro de un bloque de texto con espacio delimitado obliga a una argumentación concisa y basada en hechos relevantes. Se debe adaptar el gráfico de cada hoja para transmitir la información de forma eficaz.

- DC13 Instrumental específica:

El trabajo con el software matemático y el simulador faculta al alumno para trabajar con herramientas profesionales de alto nivel.

\subsection{Evaluación de los resultados}

Para el curso 2014-15 se obtuvo un 90\% de aprobados, superior a las medias de plan antiguo (75\%) y de Grado (78\%). Si bien, por no existir un histórico de datos, no se pueden establecer resultados de forma categórica. En cambio, la buena opinión de los alumnos sobre la metodología sí parece justificable estadísticamente. La Tabla 3 recoge los resultados referentes a las preguntas más relacionadas con la adquisición de competencias de una encuesta realizada a todos los alumnos en el siguiente cuatrimestre.

Tabla 3. Resultados de encuesta al alumnado ( $\mathrm{N}=30$ alumnos)

\begin{tabular}{lc}
\hline \multicolumn{1}{c}{ Pregunta } & \%Acuerdo \\
\hline Considero adecuado favorecer el análisis crítico en el Máster & $75.0 \%$ \\
Los conocimientos adquiridos son importantes para mi currículo & $82.6 \%$ \\
El uso de Mathcad me capacita para resolver problemas complejos & $91.7 \%$ \\
Las prácticas ayudan a consolidar la teoría & $57.1 \%$ \\
Considero adecuado que los problemas sean realistas & $91.7 \%$ \\
Considero adecuado que haya distinta metodología en Grado y en Máster & $66.7 \%$ \\
\hline
\end{tabular}

(c)) EY-NC-ND 2015, Universitat Politècnica de València 


\section{Conclusiones}

La utilización de problemas más abiertos junto con herramientas de cálculo y simulación permite desarrollar tanto competencias asociadas a la realización de problemas como instrumentales. Proporcionar una parte de los cálculos, permite además una ganancia de tiempo para poder explorar distintas soluciones y desarrollar competencias adicionales.

Respecto de planes anteriores, el rendimiento del alumnado aumentó, así como la asistencia a prácticas que fue casi del $100 \%$. Asimismo, las encuestas revelaron que una mayoría de alumnos consideraba que debía existir una metodología docente diferente en la clase de problemas para Grado y para Máster.

Consideramos además que el enfoque metodológico expuesto puede extrapolarse a otras asignaturas técnicas.

\section{Agradecimientos}

Los autores agradecen al Vicerrectorado de Estudios y Convergencia Europea y al Instituto de Ciencias de Educación de la Universitat Politècnica de València la concesión del Proyecto de Innovación y Mejora Educativa PIME A26/14 "Desarrollo de estrategias para la mejora del aprendizaje y evaluación de los problemas de Operaciones de Separación"

\section{Referencias}

GozÁlvez Zafrilla, J.M., SAntafé Moros, A. y Lora García, J. (2012). Cálculo de operaciones de separación por etapas. Valencia: Editorial Universitat Politècnica de Valencia

GozÁlvez Zafrilla, J.M., SANTAfé Moros, A. (2008) “Enseñanza de la simulación de procesos de membrana mediante objetos de aprendizaje y herramientas de cálculo”. $V$ Congreso Iberoamericano de Docencia Universitaria. Valencia

Molero, M., SAlvador, A (2014) “Resolución de problemas. Estrategias heurísticas” < http://www2.caminos.upm.es/departamentos/matematicas/Fdistancia/PIE/Problemas/ESTRATEG IAS\%20HEUR\%C3\%8DSTICAS.pdf> [Consulta: 20 de enero de 2015]

Perry, R.H. (2008) Chemical Engineers' Handbook, 8 a Ed., New York: McGraw-Hill

SAntafÉ, A., GozÁlvez, J.M. (2009) “Objetos de aprendizaje (localizables en buscador)” $<$ http://riunet.upv.es/discover> [Consulta: 20 de mayo de 2015]

Santafé Moros, A., GozÁlvez Zafrilla, J.M. y J. Lora (2013). Cálculo de operaciones de separación con Mathcad. Valencia: Editorial Universitat Politècnica de València (en papel y e-book)

VECA [Vicerrectorado de Estudios Calidad y Acreditación de la UPV] (2014). Documento marco UPV de definición de dimensiones competenciales. Universitat Politècnica de Valencia.

\section{(cc) EY-NC-ND 2015, Universitat Politècnica de València}

Congreso In-Red (2015) 